\title{
Adherence to treatment guidelines in clinical practice: study of antipsychotic treatment prior to clozapine initiation ${ }^{\dagger}$
}

\author{
Oliver D. Howes, ${ }^{*}$ Francis Vergunst, ${ }^{*}$ Siobhan Gee, Philip McGuire, Shitij Kapur \\ and David Taylor
}

\section{Background}

Clozapine is the only antipsychotic drug licensed for treatment-resistant schizophrenia but its use is often delayed. Since previous studies, national guidelines on the use of clozapine and other antipsychotics have been disseminated to clinicians.

\section{Aims}

To determine the theoretical delay to clozapine initiation and to quantify the prior use of antipsychotic polypharmacy and high-dose antipsychotic treatment.

\section{Method}

Clinico-demographic data were extracted from the treatment records of all patients commencing clozapine in our centre between 2006 and 2010 .

\section{Results}

Complete records were available for 149 patients. The mean theoretical delay in initiating clozapine was 47.7 months (s.d. =49.7). Before commencing clozapine, antipsychotic polypharmacy and high-dose treatment was evident in 36.2 and $34.2 \%$ of patients respectively. Theoretical delay was related to illness duration ( $\beta=0.7, P<0.001)$ but did not differ by gender or ethnicity.

\section{Conclusions}

Substantial delays to clozapine initiation remain and antipsychotic polypharmacy and high doses are commonly used prior to clozapine, despite treatment guidelines.

\section{Declaration of interest}

D.T. has received consultancies fees, lecturing honoraria and/or research funding from AstraZeneca, Janssen-Cilag, Servier, Sanofi-Aventis, Lundbeck, Bristol-Myers Squibb (BMS), Novartis, Eli Lilly and Wyeth. O.D.H. has been a speaker at meetings organised by and/or received investigatorinitiated charitable research funding from Astra-Zeneca, BMS, Eli Lilly, and Jansenn-Cilag. S.K. has received grant support from AstraZeneca and GSK, and has served as consultant and/or speaker for AstraZeneca, Bioline, BMS-Otsuka, Eli Lilly, Janssen (J\&J), Lundbeck, NeuroSearch, Pfizer, Roche, Servier and Solvay Wyeth.
Schizophrenia is treatment resistant in about one in three patients. ${ }^{1-4}$ This has been defined as an inadequate response to sequential treatment with two different antipsychotics at adequate dose, duration and adherence. ${ }^{3,4}$ Clozapine is the only drug treatment currently licensed for patients with treatment-resistant schizophrenia, and is associated with lower rates of re-admittance to hospital compared with other antipsychotics. ${ }^{4,5}$ However, long delays in initiating clozapine in routine clinical practice have been reported. ${ }^{6-9}$ Since our previous study, ${ }^{8}$ clinical guidelines from the National Institute for Health and Clinical Excellence (NICE) and other organisations have been published recommending that clozapine be offered at the earliest opportunity for patients with treatment-resistant schizophrenia. ${ }^{10,11}$ Although there is little clear benefit of clozapine as a first-line treatment, ${ }^{12}$ a study of patients presenting to services for the first time found that $75 \%$ of patients who failed to respond to sequential 4-week treatment trials with two different antipsychotics then responded to clozapine. ${ }^{13}$

Guidelines issued by NICE and other organisations also state that there is little evidence to support the use of antipsychotic doses above the licensed maximum dose or for antipsychotic polypharmacy (other than for short periods during cross-tapering) and recommend that these strategies should be used only in exceptional cases. ${ }^{10,11}$ The aim of our current study was to determine whether prescribing practice follows NICE clinical guidelines. Specifically, we sought to determine the time taken to initiate clozapine after a patient had completed adequate

*These authors contributed equally to the work

†See editorial, pp. 425-427, this issue. treatment with two different antipsychotic drugs, and the frequency of antipsychotic polypharmacy and high-dose antipsychotic treatment prior to clozapine initiation.

\section{Method}

The study included all patients identified using the clozapine monitoring system who commenced clozapine for the first time from 1 January 2006 to 15 April 2010 in the hospital or outpatient services, including tertiary services $(<50$ patients with schizophrenia), at the South London and Maudsley NHS Foundation Trust. The study was approved by the Trust's Drugs and Therapeutics Committee.

The following data were extracted from the clinical records: the primary diagnosis (according to ICD-10 criteria), ${ }^{14}$ selfreported ethnicity (categorised as White British/other, Black British/other, Asian British/other, or mixed); duration of illness (defined as the time from the first recording of the diagnosis of a psychotic illness by a clinician to the present); and treatment history. An 'antipsychotic treatment episode' was defined as the prescription of a regular daily dose of an antipsychotic at any dose for at least $24 \mathrm{~h}$. Antipsychotic drugs prescribed 'as required' were not counted. An adequate treatment episode was defined as an antipsychotic prescribed regularly (defined as above) at or above the minimum therapeutic dose (according to the British National Formulary $(\mathrm{BNF}))^{15}$ given the patient's age and dosing schedule for at least 6 weeks in line with the NICE guidelines. Adequate use of the same drug at different times was recorded as multiple 
episodes. Where treatment adherence was noted to be poor, the episode was counted as inadequate.

Information on antipsychotic treatment was extracted as follows:

(a) the total number of antipsychotic treatment episodes prior to the first use of clozapine (where the same antipsychotic was given on more than one occasion separated by at least 6 weeks, and each occasion met the treatment episode criteria, these were counted as separate episodes);

(b) the number of different antipsychotic drugs used (as treatment episodes) before the first use of clozapine;

(c) the number of adequate antipsychotic treatment episodes before first clozapine use;

(d) the number of adequate treatment episodes of different antipsychotics before the first use of clozapine using the same definition of adequate treatment as above and with no double counting of drugs;

(e) the number of antipsychotic treatment episodes with different second-generation drugs before first starting clozapine. Secondgeneration antipsychotic drugs were defined as amisulpride, aripiprazole, olanzapine, quetiapine, remoxipride (now withdrawn in Europe), risperidone, sertindole, ziprasidone and zotepine, and each drug was only counted once (even if a drug had been used for more than one treatment episode).

Antipsychotic polypharmacy was defined as the regular co-prescription of two or more antipsychotics each meeting the criteria for a treatment episode as above (drugs prescribed 'as required' or given irregularly were not included). Treatment episodes where the antipsychotic dose was above the maximum therapeutic dose or below the minimum therapeutic (subtherapeutic) dose given in the BNF were recorded separately (in the case of polypharmacy this was determined from the combined total dose, calculated as described in the Maudsley Prescribing Guidelines). ${ }^{4,15}$

The primary outcome measure was the maximum theoretical delay in clozapine initiation. This was determined in patients who had received adequate antipsychotic treatment episodes (as defined above) with at least two different antipsychotic drugs (the point at which their illness could first meet NICE criteria for treatment resistance) and was defined as the time from the end of the second adequate antipsychotic treatment episode to the first clozapine use. Thus, a patient who completed the second adequate antipsychotic treatment episode in January 2005 and who started clozapine in January 2006 would have a theoretical delay of 1 year. The period before January 1990 was excluded as clozapine was not available in the UK before then. In cases where a patient's first documented antipsychotic prescription involved polypharmacy, each additional adequate antipsychotic trial was counted as an additional treatment episode. Where the adequacy of a treatment episode could not be determined because information on dose or duration was missing, this episode was excluded from the analyses of adequate trials but it was counted for the purposes of total number of antipsychotic treatment episodes.

\section{Statistical analysis}

After determining whether variances were equal and conformed to a normal distribution using Levene's test and the Kolmorogov-Smirnov Z-test respectively, two-tailed MannWitney $U$ - or Kruskal-Wallis tests were used as appropriate to test whether there were differences in theoretical delay by gender, diagnostic or ethnic group. In an exploratory analysis, the relationship between theoretical delay (dependent variable) and age and illness duration (independent variables) was determined using regression.

\section{Results}

We identified 227 patients who commenced clozapine for the first time between 1 January 2006 and 15 April 2010. Of these, 78 (34.4\%) were excluded from subsequent analyses because notes were missing, and so a complete prescribing history was not available. The demographic and clinical characteristics of the included and excluded individuals are shown in Table 1. There were significant differences in age, gender, ethnicity, diagnosis and duration of illness between the two groups, with the included participants tending to be younger, to have shorter duration of illness, more likely to be male, diagnosed with schizophrenia and be from a Black, Asian or mixed ethnic group (Table 1).

Of the 149 individuals included in the study, $68.5 \%$ were male, $79.9 \%$ were diagnosed with schizophrenia, $18.1 \%$ with schizoaffective disorder, $1.3 \%$ with bipolar disorder and $0.7 \%$ with psychotic depression. In total, 132 participants (88.6\%) were started on clozapine for the first time as in-patients and 17 $(11.4 \%)$ started it in the community. The range of duration of illness was $1.8-30$ years.

\begin{tabular}{|c|c|c|c|c|c|}
\hline & Included & Excluded & $t$-test (d.f.) & $Z$-test & $P$ \\
\hline Age, years: mean (s.d.) & $34.0(10.2)$ & $43.1(12.6)$ & $5.4(124)$ & & $<0.0001$ \\
\hline Gender, $n$ & & & & 2.2 & 0.027 \\
\hline Male & 102 & 40 & & & \\
\hline Female & 47 & 35 & & & \\
\hline Ethnicity, $n$ & & & & 2.5 & 0.013 \\
\hline White & 61 & 45 & & & \\
\hline Black & 61 & 20 & & & \\
\hline Asian & 7 & 5 & & & \\
\hline Mixed & 12 & 2 & & & \\
\hline Other & 8 & 0 & & & \\
\hline Diagnosis, $n$ & & & & 2.6 & 0.01 \\
\hline Schizophrenia & 119 & 50 & & & \\
\hline Schizoaffective disorder & 27 & 13 & & & \\
\hline Bipolar disorder & 2 & 7 & & & \\
\hline Other & 1 & 5 & & & \\
\hline Duration of illness, years & & & & 6.7 & 0.0001 \\
\hline Mean (s.d.) & $8.6(5.4)$ & $16.4(9.2)$ & & & \\
\hline Median (interquartile range) & $7(6)$ & $15(12)$ & & & \\
\hline
\end{tabular}


There were a total of 825 antipsychotic treatment episodes recorded for the participants included in the study. A total of 91 $(11.0 \%)$ episodes could not be evaluated due to incomplete information (either the dose or duration was missing for some time points). Of the remaining 734 episodes, 189 (25.7\%) were inadequate, either because they used a subtherapeutic dose $(n=83$, $11.3 \%)$ or were too short in duration $(n=88,12.0 \%)$ or were both at a subtherapeutic dose and too short in duration $(n=18,2.5 \%)$.

The antipsychotic treatment history preceding clozapine is summarised in Table 2. Before commencing clozapine, 143 patients $(96.0 \%)$ received at least one adequate trial of a secondgeneration antipsychotic, and 85 patients $(57 \%)$ had received a depot antipsychotic. Polypharmacy was evident in 54 patients $(36.2 \%)$. A total of 51 patients (34.2\%) had received antipsychotic treatment above the maximum licensed dose $(45.9 \%$ during monotherapy, $54.1 \%$ during polypharmacy).

In total 95 patients $(63.8 \%)$ were switched directly from an oral antipsychotic medication to clozapine. Of these, 86 (90.5\%) were switched from a second-generation drug, $8(8.4 \%)$ from a firstgeneration drug and $1(1.1 \%)$ from a combination of a first- and a second-generation drug. Also, 34 patients $(22.8 \%)$ were switched directly from depot medication, $16(10.7 \%)$ from a combination of a depot and oral medication, and $4(2.7 \%)$ had received no treatment for at least 1 month prior to commencing clozapine.

Before commencing clozapine, 129 patients $(86.6 \%)$ had received at least two adequate trials of different antipsychotics. The remaining $20(13.4 \%)$ had not received two adequate antipsychotic treatment episodes $(18(12.1 \%)$ had received one and $2(1.3 \%)$ had not received any adequate treatment trials) before commencing clozapine. The mean theoretical delay in the 129 individuals who had received two adequate treatment trials was 47.7 (s.d. $=49.7$ ) months (median 32 (interquartile range, $\mathrm{IQR}=58)$, range 0-219).

There was a significant relationship between theoretical delay and age $(\beta=0.3, t=3.5, P=0.001)$, and between delay and illness duration $(\beta=0.7, t=12.5, P<0.001$, Fig. 1 , where theoretical delay (months) was $16+0.7 \times$ illness duration (months)). However, the relationship between age and delay was not significant after adjusting for illness duration ( $\beta=0.02, t=0.3, P=0.8)$. There was no significant difference in theoretical delay between female and male patients (female: mean 64.8 months (s.d. $=64$ ), median 46.5 (IQR =93); male: mean 39.5 months (s.d.=39), median 29 $(\mathrm{IQR}=43) ; Z=1.5, \quad P=0.13)$ or between diagnostic groups (schizophrenia: mean 47.5 months (s.d.=52.4), median 29 $(\mathrm{IQR}=57)$; any other diagnosis: mean 48.6 months (s.d.=39.5), median 41 (IQR $=70) ; Z=0.8, P=0.4$ ), or ethnicity (White: mean 48.3 months (s.d. $=53)$, median $31.5(\mathrm{IQR}=57)$; other: mean 47.4 months (s.d. $=47.9)$, median $32(\mathrm{IQR}=59) ; Z=0.2, P=0.9)$.

\section{Discussion}

This study had two main findings. First, that in routine clinical practice, clozapine initiation was delayed by a mean of 4 years,

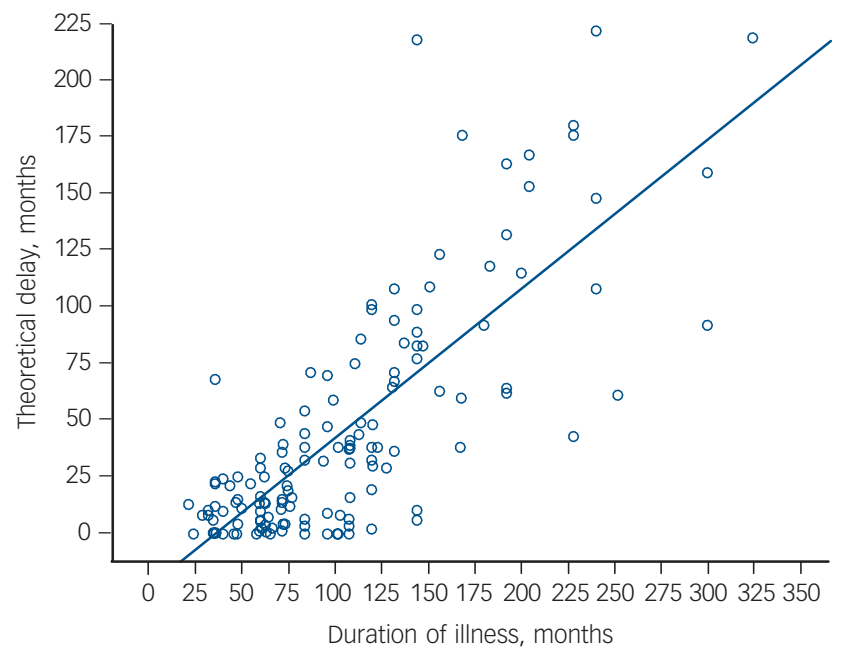

Fig. 1 The relationship between theoretical delay to clozapine initiation and illness duration $(\beta=0.7, P<0.001)$.

and second, that other antipsychotics were often used instead, in ways not recommended by therapeutic guidelines. Thus, prior to commencing clozapine, over a third of patients had received antipsychotic polypharmacy, and over a third had received antipsychotic treatment above the licensed maximum dose, even though there is little evidence to support either strategy, and both are associated with increased risks of side-effects and complications. ${ }^{4,10,16,17}$ Prior to commencing clozapine, patients received, on average, more than five different antipsychotic treatment episodes. About 50 patients started clozapine each year. Given that there are over 5000 patients with schizophrenia in the Trust and about 350 new patients present each year (details available from the authors on request), this suggests that clozapine is underused.

\section{Methodological considerations}

One potential limitation is that we did not determine the reason for initiating clozapine. It is possible that some of the patients commencing clozapine were doing so for treatment intolerance rather than treatment resistance. However, a number of secondgeneration antipsychotics have been available since the mid-1990s as alternatives to clozapine for treatment intolerance. ${ }^{4,18-20}$ As $96 \%$ of the patients had received at least one second-generation antipsychotic, it is thus unlikely that antipsychotic intolerance was the reason for many, if any, of the patients commencing clozapine. A further potential limitation is that we did not determine the response to prior antipsychotic treatment. It is thus possible that some patients initially showed a good response but developed treatment resistance later. Although prospective

\begin{tabular}{|c|c|c|c|}
\hline Treatment, $n$ & Mean (s.d.) & Median (IQR) & Range \\
\hline Total antipsychotic treatment episodes & $5.6(3.5)$ & $5(4)$ & $1-20$ \\
\hline Total different antipsychotic drugs given & $3.9(1.9)$ & $3(3)$ & $1-10$ \\
\hline \multicolumn{4}{|l|}{ Total adequate treatment episodes } \\
\hline Any antipsychotic & $3.7(2.4)$ & $3(3)$ & $0-12$ \\
\hline Different drugs & $2.8(1.5)$ & $2(2)$ & $0-6$ \\
\hline Total different second-generation antipsychotics drugs used & $2.5(1.0)$ & $2(1)$ & $0-5$ \\
\hline Different second-generation antipsychotics treatment episodes & $2.0(0.9)$ & $2(2)$ & $0-4$ \\
\hline
\end{tabular}


studies are needed to definitively test this, for it to account for the long time to initiate clozapine, patients would have been treated on one drug for a long time, and then, as treatment resistance developed, switched to another drug before starting clozapine within a relatively short period of time. However, this pattern of treatment was not evident - instead they received multiple treatment episodes and different antipsychotic drugs. Although we excluded treatment episodes where adherence was noted to be poor, another limitation of the study is that some adequate trials may in fact have been inadequate due to covert non-adherence.

Our data on antipsychotic high doses and polypharmacy were restricted to regular prescriptions: the rates of both would have been higher if we had included 'as required' prescriptions as well. A number of patients had to be excluded from the study because complete treatment histories were not available and this may limit the generalisability of our findings. As the excluded patients had significantly longer illness durations than the included patients, and greater illness duration was associated with greater initiation delay, the delay in initiating clozapine may be longer if we had been able to include all patients. A total of 91 (11\%) treatment episodes were not included because of missing data and this means there is some imprecision in our data. For example, if all of these episodes had been inadequate this would have increased the percentage of inadequate episodes by $8.2 \%$ (from 25.7 to $33.9 \%$ ), and reduced the percentage of adequate episodes by the same amount. Finally, our study is limited to patients who have started clozapine - the treatment of refractory patients who have not started clozapine remains to be determined.

\section{Implications of our findings}

In comparison with the mean delay of 5 years in our 2002 study in the same setting, ${ }^{8}$ the delay to clozapine initiation in our current study was shorter but remained substantial despite the publication of the NICE guidelines in 2002. ${ }^{11}$ As the guidelines were disseminated to all clinicians, unlike in 2002, it is not possible to argue that lack of familiarity with the recommendations or the evidence underlying them, underlies these findings. Furthermore, it is also clear from guidelines published since then that the consensus on these issues did not change during our study period. ${ }^{3,10,21}$ Our current findings, thus, suggest that schizophrenia treatment guidelines have had little impact on the time taken to initiate clozapine, or on the use of high-dose antipsychotics or polypharmacy prior to clozapine in this setting, although it could be argued that we would see greater effects if the study was restricted to patients whose first antipsychotic treatment began after the publication of the guidelines. The mean number of different adequate antipsychotic treatment episodes was 2.8, lower than in our previous study (four drugs) ${ }^{8}-$ suggesting that this aspect of prescribing is closer to guidelines now.

Coupled with evidence of the underuse of clozapine in many settings, ${ }^{22}$ the long delay in initiating clozapine and also the common use of treatments that guidelines recommend are restricted to exceptional cases, our study suggests that there are major barriers to clinicians initiating clozapine. Patient-related factors, such as refusing blood monitoring, are likely to contribute to clozapine's underuse and delays in initiation, although it is noteworthy that once patients are on clozapine the vast majority report its advantages outweigh its disadvantages. ${ }^{23,24}$ Furthermore, although patient factors are unlikely to vary markedly between settings, the use of clozapine varies greatly across settings and countries $9,25,26$ and organisational changes have been associated with a 2.9 -year reduction in the mean delay to initiate clozapine. $^{6}$ This suggests that organisational- and clinician-related factors have a major impact and are potentially modifiable contributors to the delay we observed.

Clozapine has a complex dose titration regime and requires daily monitoring during the initiation phase and careful management of treatment emergent side-effects. Many clinicians have few patients on clozapine under their care, and are less familiar with managing the initiation of clozapine than other antipsychotic drugs. ${ }^{27}$ These factors may contribute to the delay we observed. However, it is possible to initiate clozapine quickly for individuals whose condition is treatment resistant where resources are available, ${ }^{13}$ suggesting that the availability of additional resources and expertise might reduce delays in clozapine initiation.

In summary, in routine practice there is often a long delay before the use of clozapine. Moreover, during this period, other antipsychotics are commonly used in higher than licensed doses, or in combination, despite the lack of evidence to support these strategies.

Oliver D. Howes, MRCPsych, PhD, Department of Psychosis Studies, Institute of Psychiatry, London, and South London and Maudsley NHS Foundation Trust, London; Francis Vergunst, BSc, Department of Psychosis Studies, Institute of Psychiatry, London; Siobhan Gee, MPharm, PGDipGPP, MRPharmS, Pharmacy Department, South London and Maudsley NHS Foundation Trust, London; Philip McGuire FRCPsych, PhD, Shitij Kapur, MBBS, FRCPC, PhD, Department of Psychosis Studies, Institute of Psychiatry, London, and South London and Maudsley NHS Foundation Trust, London; David Taylor, MSC, PhD, MRPharmS, Pharmacy Department, South London and Maudsley NHS Foundation Trust, London, and Institute of Pharmaceutical Sciences, King's College London, UK

Correspondence: Oliver D. Howes, Box 067, Institute of Psychiatry, De Crespigny Park, London SE5 8AF, UK. Email: oliver.howes@kcl.ac.uk

First received 7 Nov 2011, final revision 11 May 2012, accepted 29 May 2012

\section{Funding}

This study was funded by an MRC, UK grant to O.D.H. (grant code MC-A656-5QD30) and the National Institute of Health Research Biomedical Research Centre.

\section{References}

1 Mortimer AM, Singh P, Shepherd CJ, Puthiryackal J. Clozapine for treatmentresistant schizophrenia: National Institute of Clinical Excellence (NICE) guidance in the real world. Clin Schizophr Relat Psychoses 2010; 4: 49-55.

2 Lieberman JA, Kane JM, Johns CA. Clozapine: guidelines for clinical management. J Clin Psychiatry 1989; 50: 329-38.

3 National Collaborating Centre for Mental Health. Schizophrenia: Core Interventions in the Treatment and Management of Schizophrenia in Adults in Primary and Secondary Care (Updated edn). National Clinical Guideline 82. British Psychological Society and the Royal College of Psychiatrists, 2010.

4 Taylor D, Paton C, Kapur S. The Maudsley Prescribing Guidelines (10th edn). Informa Healthcare, 2010.

5 Tiihonen J, Haukka J, Taylor M, Haddad PM, Patel MX, Korhonen P. A nationwide cohort study of oral and depot antipsychotics after first hospitalization for schizophrenia. Am J Psychiatry 2011; 168: 603-9.

6 Harrison J, Janlov M, Wheeler AJ. Patterns of clozapine prescribing in a mental health service in New Zealand. Pharm World Sci 2010; 32: 503-11.

7 Kelly DL, Kreyenbuhl J, Dixon L, Love RC, Medoff D, Conley RR. Clozapine underutilization and discontinuation in African Americans due to leucopenia. Schizophr Bull 2007; 33: 1221-4.

8 Taylor DM, Young C, Paton C. Prior antipsychotic prescribing in patients currently receiving clozapine: a case note review. J Clin Psychiatry 2003; 64: $30-4$

9 Wheeler AJ. Treatment pathway and patterns of clozapine prescribing for schizophrenia in New Zealand. Ann Pharmacother 2008; 42: 852-60.

10 Barnes TR. Evidence-based guidelines for the pharmacological treatment of schizophrenia: recommendations from the British Association for Psychopharmacology. J Psychopharmacol 2011; 25: 567-620. 
11 National Institute for Health and Clinical Excellence. Schizophrenia: Core Interventions in the Treatment and Management of Schizophrenia in Primary and Secondary Care (Clinical Guideline 1). London: NICE, 2002.

12 Agid O, Remington G, Kapur S, Arenovich T, Zipursky RB. Early use of clozapine for poorly responding first-episode psychosis. J Clin Psychopharmacol 2007; 27: 369-73.

13 Agid O, Arenovich T, Sajeev G, Zipursky RB, Kapur S, Foussias G, et al. An algorithm-based approach to first-episode schizophrenia: response rates over 3 prospective antipsychotic trials with a retrospective data analysis. Clin Psychiatry 2011; 72: 1439-44.

14 World Health Organization. The ICD-10 Classification of Mental and Behavioural Disorders: Clinical Descriptions and Diagnostic Guidelines. WHO 1992.

15 Joint Formulary Committee. British National Formulary (BNF59). BMJ Group and Pharmaceutical Press, 2010.

16 Barnes TR, Paton C. Antipsychotic polypharmacy in schizophrenia: benefits and risks. CNS Drugs 2011; 25: 383-99.

17 Howes OD, Egerton A, Allan V, McGuire P, Stokes P, Kapur S. Mechanisms underlying psychosis and antipsychotic treatment response in schizophrenia: insights from PET and SPECT imaging. Curr Pharm Des 2009; 15: 2550-9.

18 Howes OD, Bhatnagar A, Gaughran FP, Amiel SA, Murray RM, Pilowsky LS. A prospective study of impairment in glucose control caused by clozapine without changes in insulin resistance. Am J Psychiatry 2004; 161: 361-3.
19 Howes OD, Wheeler MJ, Meaney AM, O'Keane V, Fogelman I, Blake G, et al. Bone mineral density and its relationship to prolactin levels in patients taking antipsychotic treatment. J Clin Psychopharmacol 2005; 25: 259-61.

20 Howes OD, Wheeler MJ, Pilowsky LS, Landau S, Murray RM, Smith S. Sexual function and gonadal hormones in patients taking antipsychotic treatment for schizophrenia or schizoaffective disorder. J Clin Psychiatry 2007; 68: 361-7.

21 Buchanan RW, Kreyenbuhl J, Kelly DL, Noel JM, Boggs DL, Fischer BA, et al. The 2009 schizophrenia PORT psychopharmacological treatment recommendations and summary statements. Schizophr Bull 2010; 36: 71-93.

22 Kelly DL, Dixon LB, Kreyenbuhl JA, Medoff D, Lehman AF, Love RC, et al. Clozapine utilization and outcomes by race in a public mental health system: 1994-2000. J Clin Psychiatry 2006; 67: 1404-11.

23 Taylor D, Shapland L, Laverick G, Bond J, Munro J. Clozapine: a survey of patient perceptions. Psychiatr Bull 2000; 24: 450-2.

24 Angermeyer MC, Loffler W, Muller P, Schulze B, Priebe S. Patients' and relatives' assessment of clozapine treatment. Psychol Med 2001; 31: 509-17.

25 Downs J, Zinkler M. Clozapine: national review of postcode prescribing. Psychiatr Bull 2007; 31: 384-7.

26 Tang YL, Mao PX, Jiang F, Chen Q, Wang CY, Cai ZJ, et al. Clozapine in China. Pharmacopsychiatry 2008; 41: 1-9.

27 Nielsen J, Dahm M, Lublin H, Taylor D. Psychiatrists' attitude towards and knowledge of clozapine treatment. J Psychopharmacol 2010; 24: 965-71.

\section{poems by doctors}

\section{Bitter Treatment}

\section{Sandy Goldbeck-Wood}

it was the area between

the midlines of the two

temporal lobes, and back

for eight to nine centimetres -

the hippocampus, the parahippocampal

gyrus, the entorhinal and perirhinal

cortices, the amygdala -

the young man was awake

on the table the whole time, apparently

as Dr Scoville inserted a silver straw

and sucked out nearly the entire

greyish-pink mass including

his memory, like finest mate amargo

through a bombilla.

This poem is from The Hippocrates Prize 2011, published by The Hippocrates Prize in association with Top Edge Press. (c) Sandy Goldbeck-Wood.

Chosen by Femi Oyebode.
The British Journal of Psychiatry (2012)

201, 485. doi: 10.1192/bjp.bp.111.103093 